\title{
Pregabalin in Neuropathic Pain Related to DPN, Cancer and Back Pain: Analysis of a 6-Week Observational Study
}

\author{
Thomas R. Toelle ${ }^{1}$, Roxana Varvara ${ }^{2},{ }^{*}$, Meryem Nimour $^{2}$, Birol Emir $^{3}$ and Matthias Brasser ${ }^{4}$ \\ ${ }^{I}$ Interdisciplinary Pain Clinic, Munich Technical University, Munich, Germany \\ ${ }^{2}$ Pfizer Ltd, Surrey, UK \\ ${ }^{3}$ Pfizer Inc, New York, NY, USA \\ ${ }^{4}$ Pfizer Pharma GmbH, Berlin, Germany
}

\begin{abstract}
Background: Neuropathic pain is associated with many conditions. Pregabalin has demonstrated efficacy in randomized, controlled trials (RCTs) in peripheral and central neuropathic pain. Observational studies complement findings from RCTs by enabling an agent to be studied in real-world patients and circumstances.

Methods: Patients with neuropathic pain were treated with pregabalin 150-600 mg/day in this 6 week observational study. Analyses of subsets of patients with painful diabetic peripheral neuropathy (DPN; $n=4633$ ), back pain with a neuropathic component $(n=3800)$, and cancer-related neuropathic pain $(n=345)$ were undertaken.

Results: The mean pregabalin doses ranged from 219 to $250 \mathrm{mg} /$ day across the disease groups. Mean baseline pain scores (6.4 to 7.0 across the three disease states) indicated patients had moderate to severe pain. Pregabalin was associated with a rapid and significant reduction in pain from week 1 to endpoint in all groups. Over $80 \%$ in each of the groups had a $\geq 30 \%$ pain reduction in their pain at 6 weeks, and over two-thirds had a $\geq 50 \%$ reduction. Pain-related sleep interference decreased rapidly and significantly. Most patients $(87 \%)$ were either very satisfied or satisfied with the action of pregabalin. General well-being improved significantly over the 6 weeks. Pregabalin was generally well tolerated; the most common adverse event overall was dizziness (1.4\%). Few patients ( $\leq 6.1 \%$ /group) discontinued due to adverse events.

Conclusions: In neuropathic pain patients in day-to-day practice, pregabalin was associated with notable reductions in pain and sleep interference. The benefits of pregabalin were reflected in the high level of patient satisfaction and improvement in general well-being.
\end{abstract}

Keywords: Pregabalin, Back pain, DPN, Cancer, Neuropathic pain, Observational.

\section{INTRODUCTION}

Neuropathic pain was initially defined as pain initiated or caused by a primary lesion or dysfunction in the nervous system [1]. More recently it has been proposed that neuropathic pain be defined as being pain arising as a direct consequence of a lesion or disease affecting the somatosensory system either at peripheral or central level [2]. It is estimated that as many as $7-8 \%$ of the European general population may experience neuropathic pain $[3,4]$. Neuropathic pain is associated with many conditions including metabolic disorders such as diabetes, surgery, nerve entrapment, conditions causing central lesions such as stroke, and with infections, as in the case of post-herpetic neuralgia [5]. In conditions such as radiculopathy (sciatica) and cancer-related pain, the pain patients experience may have both neuropathic and nociceptive components, sometimes referred to as "mixed pain" [6-8]. Neuropathic

*Address correspondence to this author at Pfizer Ltd, Walton Oaks, Dorking Road, Tadworth, Surrey, KT20 7NS, UK; Tel: +44 (0) 1737331 904; Fax: +44 (0) 1737332 214; E-mail: Roxana.Varvara@Pfizer.com pain is debilitating, costly and does not respond to conventional analgesics [5].

Pregabalin 150-600 mg/day has been shown to be effective at relieving pain and reducing pain-related sleep interference in numerous well controlled trials of patients with painful diabetic peripheral neuropathy (DPN) [9] and post-herpetic neuralgia $[10,11]$, in a trial in post-traumatic peripheral neuropathic pain [12], and in a trial in central neuropathic pain associated with spinal cord injury [13]. In addition to improving pain-related sleep interference, pregabalin has also shown to improve composite indices of sleep disturbance in patients with neuropathic pain assessed using the Medical Outcomes Sleep Scale [12, 13]. Pregabalin is also effective in relieving pain associated with fibromyalgia [14], in the treatment of generalized anxiety disorder [15] and in partial onset seizures [16].

The efficacy of pregabalin across various diseases, including neuropathic pain, has been attributed to the modulation of neurotransmitter release from hyperexcited neurons via binding to the alpha-2-delta subunit, which modulates calcium influx into the pre-synaptic terminal [17, 18]. The effect of pregabalin in neuropathic pain is also 
linked to impaired anterograde trafficking of alpha-2-delta-1, resulting in its decrease in presynaptic terminals, which is also thought to reduce neurotransmitter release and spinal sensitization, an important factor in the maintenance of neuropathic pain [19]. It has also been shown to cause supraspinal activation of noradrenergic descending inhibition to reduce neuropathic pain [20]. Pregabalin has linear, predictable pharmacokinetics and, unlike gabapentin, its absorption is not saturable [21]. Pregabalin is neither bound to plasma proteins nor hepatically metabolized [21], and thus is not subject to any known pharmacokinetic drug-drug interactions $[21,22]$. The pharmacokinetic and drug interaction profile makes pregabalin quite a straight forward agent to use in conditions such as neuropathic pain where polypharmacy is often required, both for pain management and for the management of concurrent medical conditions.

Pregabalin is recommended as a first-line treatment for neuropathic pain by the Neuropathic Pain Special Interest Group (NeuPSIG) of the International Association for the Study of Pain [23]. It is also recommended as a first-line treatment for DPN, PHN and central neuropathic pain by the European Federation of Neurological Societies (EFNS) [24] and as first-line treatment for DPN by the American Academy of Neurology [25]. Pregabalin is also recommended as an adjuvant in cancer pain [26, 27]. It is licensed for adults in Europe for the treatment of peripheral and central neuropathic pain, in addition to GAD and as adjunctive treatment of partial seizures [28]. Thus, in clinical practice pregabalin is used in a wide variety of patients with neuropathic pain of a wide variety of etiologies. While randomized, placebo-controlled, double-blind trials (RCTs) provide the level of rigour needed to generate reliable evidence about the efficacy and tolerability of a drug treatment, patients included in RCTs are not always representative of the broader range of patients encountered in real-life clinical practice. Observational studies in day-to-day clinical practice are useful to provide additional insight into how an agent behaves in the real world, and how generalizable the findings from RCTs might be.

Here we report the results of a post-hoc analysis of a large, 6-week observational study of pregabalin in the treatment of neuropathic pain in usual clinical care that has been reported elsew here [29]. From the large database created in the execution of this study we analyzed data from subsets of patients with DPN, back pain with a neuropathic component, and cancer-related neuropathic pain. The previous report by Mallison included data on a pain endpoint in patients according to their primary aetiologies, but no other endpoints according to aetiology. We were particularly interested gaining further insight into the effects of pregabalin in patients with DPN, back pain and cancer pain, without other concomitant neuropathic pain states, as they represent three common and troublesome pain conditions. Back pain with a neuropathic component and cancer pain are usually not "pure" neuropathic pain states, often being a combination of neuropathic and nociceptive pain, and thus present considerable challenges to clinicians. This analysis provided the opportunity to explore beyond just the effects on pain itself as assessments of patient-centred outcomes such as sleep, well-being, and satisfaction were also included.

\section{MATERIALS AND METHODS}

\section{Patients}

Adult patients with peripheral neuropathic pain, with a diagnosis based on clinical opinion, of any etiology were allowed to take part. The inclusion of patients was based entirely on clinical opinion and whatever diagnostic procedures the physician would normally undertake without any specified formalised assessment to confirm the presence of the neuropathic component. Those who were already taking other analgesics for their neuropathic pain were allowed to continue doing so and to have pregabalin added to their current regimen.

\section{Study Design and Treatment}

This was a 6-week, open-label, observational study in standard outpatient settings in Germany. In total 5808 physicians were involved in the study comprising $68 \%$ general practitioners, $16 \%$ internal medicine specialists, $4 \%$ psychiatrists, $4 \%$ neurologists, $4 \%$ orthopedists, $4 \%$ anesthetists, and $2 \%$ surgeons. The physicians were free to prescribe pregabalin 150-600 mg/day as either monotherapy or add-on therapy, in accordance with the European Summary of Product Characteristics (SmPC) dosing schedule [28].

\section{ASSESSMENTS}

Patients visited the clinic for assessment at baseline, week 1 , week 3 and week 6 (or upon early discontinuation). At each visit the case report form was completed; patients did not keep daily diaries as is typically done in RCTs. At each visit patients were asked to rate their average pain intensity on an 11-point numerical rating scale (NRS) from $0=$ no pain to $10=$ worst imaginable pain. At baseline the pain characteristics were evaluated as either intermittent or persistent. The extent to which pain interfered with sleep was also rated on an 11-point NRS from 0=not impaired to $10=$ severely impaired. Patients also rated the extent of impairments in general well-being on the same NRS. To further evaluate well-being, patients were asked how often during the past week they felt "calm and relaxed", "full of energy", and "discouraged and sad", rated on a 6-point Likert scale from $1=$ always to $6=$ never. Some patients also completed the Pittsburgh Sleep Quality Index (PSQI ) questionnaire [30] at each visit. The PSQI is a self-rated questionnaire which assesses sleep quality and disturbances over a 1-month time interval. The global score is the sum of seven component scores: subjective sleep quality, sleep latency, sleep duration, habitual sleep efficiency, sleep disturbances, use of sleeping medication, and daytime dysfunction, which are derived from 19 individual items. At each visit, the level of patient satisfaction with the "action of pregabalin" and the "tolerability of pregabalin" were each rated as very satisfied, satisfied, moderately satisfied or dissatisfied. The reasons for early discontinuation were recorded and all adverse events, regardless of presumed causal relationship with pregabalin, were recorded and coded using the coding symbols for thesaurus of adverse reaction terms (WHOCODE). Adverse events were actually elicited by the physician rather than relying on spontaneous reports. The presence of depression or a sleep disorder was indicated using a check-box, but no diagnostic or symptom rating scales were 
Table 1. Patient Baseline Characteristics and Concomitant Analgesic Use According to Primary Diagnosis of Cause of Neuropathic Pain

\begin{tabular}{|c|c|c|c|}
\hline & $\underset{(n=4633)}{\text { DPN }}$ & $\begin{array}{l}\text { Back Pain with Neuropathic } \\
\text { Component }(\mathrm{n}=3800)\end{array}$ & $\begin{array}{c}\text { Cancer-related Neuropathic } \\
\text { pain }(n=345)\end{array}$ \\
\hline Women, n (\%) & $2103(46)$ & $2044(54)$ & $169(50)$ \\
\hline Age range, years & $21-95$ & $19-94$ & $24-89$ \\
\hline$\geq 65$ years, $n(\%)$ & $2343(52)$ & $1213(32)$ & $141(42)$ \\
\hline - Mean (SD) years & $4.4(4.5)$ & $3.9(4.9)$ & $2.2(3.6)$ \\
\hline - Median years & 3.6 & 2.3 & 0.9 \\
\hline Intermittent pain, $\mathbf{n}(\%)$ & $1638(42)$ & $1197(37)$ & $95(33)$ \\
\hline Persistent pain, n (\%) & $2256(58)$ & $2055(63)$ & $193(67)$ \\
\hline Sleep disorder & $1129(24)$ & $1076(28)$ & $101(29)$ \\
\hline Pregabalin monotherapy, n (\%) & $2900(63)$ & $1618(43)$ & $152(44)$ \\
\hline Concomitant analgesics (pregabalin add-on $\mathbf{T x}), \mathbf{n}(\%)$ & $1733(37)$ & $2182(57)$ & $193(56)$ \\
\hline - NSAIDS/COX-2 & $1207(26)$ & $1524(40)$ & $110(32)$ \\
\hline - Analgesics for mild or moderate pain & $457(10)$ & $607(16)$ & $62(18)$ \\
\hline - Narcotic analgesics for moderate to severe pain & $197(4)$ & $455(12)$ & $63(18)$ \\
\hline - TCA & $175(4)$ & $322(8)$ & $33(10)$ \\
\hline - SNRI & $1(0.02)$ & $2(0.05)$ & 0 \\
\hline
\end{tabular}

For some assessments occasional data were missing. The presence of anxiety, depression and sleep disorder were recorded as a check box on the case report form, not identified using diagnostic or symptom rating scales. Analgesics for mild or moderate pain include paracetamol and compound analgesics containing paracetamol and aspirin. COX-2-cyclooxygenase-2 inhibitor; NSAID - nonsteroidal anti-inflammatory drug; TCA - tricyclic antidepressant; SSRI - selective serotonin reuptake inhibitor, SNRI - serotonin noradrenalin reuptake inhibitor. Previous gabapentin treatment in the last 14 days.

employed to further evaluate the presence or severity of these conditions. Other concomitant disorders were also recorded.

\section{ANALYSIS}

All calculations and analyses were undertaken for each of the three subsets of patients according to the primary neuropathic pain etiology, DPN, back pain with neuropathic component, and cancer-related neuropathic pain. As this was a non-comparative, observational study no formal statistical analysis plan was developed. The mean changes from baseline at each weekly assessment in pain intensity and the pain-related sleep interference scores were analysed using a paired t-test to determine if the changes from baseline were statistically significant at the $\mathrm{P}<0.05$ level. The proportions of patients with mild (0-3), moderate (4-6) and severe (7-10) pain and pain-related sleep interferences were also determined from the scores at baseline and week 6 . Response rates, defined as the proportions of patients with $\mathrm{a} \geq 30 \%$ or $\geq 50 \%$ reduction from baseline to week 6 in their pain and sleep interference scores were also calculated. Patients with a baseline and a post-baseline assessment were included in the analyses. For patients who discontinued after one postbaseline assessment the last observation was carried forward (LOCF) in the analyses. The PSQI was not completed satisfactorily by all patients at all sites.

\section{RESULTS}

\section{Patients and Disposition}

In total 15,301 patients with neuropathic pain were enrolled in the study [29]. From these, patients with DPN $(n=4633)$, back pain with a neuropathic component $(n=3800)$ and cancer-related neuropathic pain $(n=345)$ were included in this post hoc analysis. The demographic and illness characteristics are shown in Table $\mathbf{1}$. The characteristics are consistent with what would be expected for the specific diseases being examined in terms of their age, and illness duration. Approximately half the sample was women. The mean age of patients ranged from 58 to 64 years across the 
Table 2. Completion Rates and Reasons for Discontinuation n (\%)

\begin{tabular}{|l|c|c|c|}
\hline & DPN $(\mathbf{n}=\mathbf{4 6 3 3})$ & Back pain with Neuropathic Component $(\mathbf{n}=\mathbf{3 8 0 0})$ & Cancer-related Neuropathic Pain $(\mathbf{n}=\mathbf{3 4 5})$ \\
\hline \hline Completed, $\mathbf{n}(\%)$ & $4129(89)$ & $3215(85)$ & $287(83)$ \\
\hline All discontinued, $\mathbf{n}(\%)$ & $504(11)$ & $585(15)$ & $58(17)$ \\
\hline Lack of efficacy, $\mathbf{n}(\%)$ & $86(1.9)$ & $130(3.4)$ & $4(1.2)$ \\
\hline Adverse event, $\mathbf{n}(\%)$ & $161(3.5)$ & $153(4.0)$ & $21(6.1)$ \\
\hline Patient defaulted, $\mathbf{n}(\%)$ & $36(0.8)$ & $37(1.0)$ & $5(1.4)$ \\
\hline Other reason $\boldsymbol{\dagger}, \mathbf{n}(\%)$ & $221(4.7)$ & $265(9.6)$ & $28(8.1)$ \\
\hline
\end{tabular}

$\dagger$ Other reason includes 1 patient who died in the DPN group, 3 in the back pain group, and 6 in the cancer pain group.

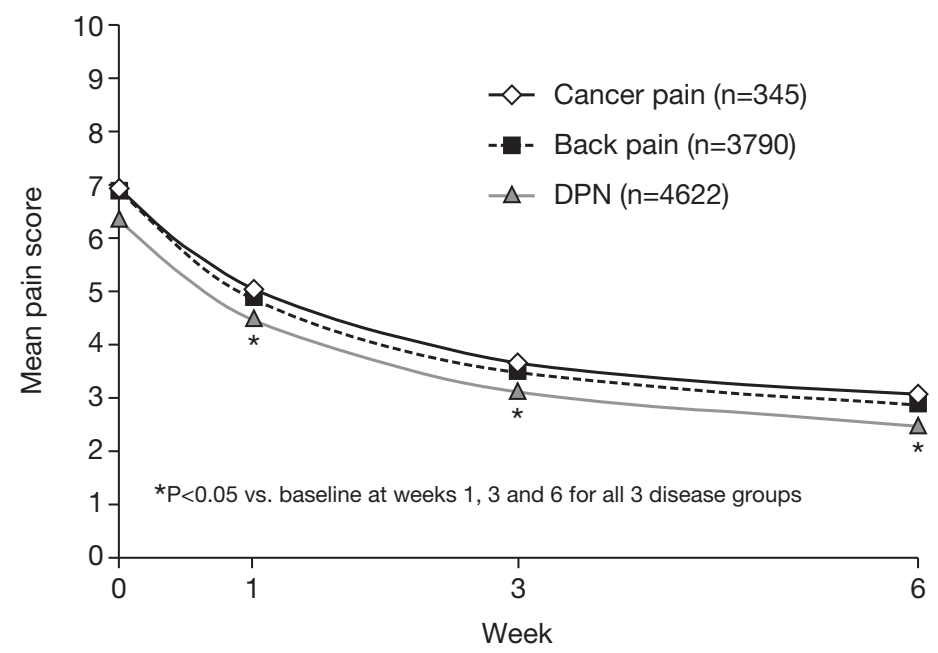

Fig. (1). Mean pain score in all patients

Each patient rated average pain intensity on an 11-point NRS from $0=$ no pain to $10=$ worst imaginable pain. $\mathrm{P}$ values for change from baseline based on paired $t$ test.

three disease groups. A notable proportion of patients in each of the three disease groups was aged at least 65 years (32$52 \%$ ) across groups. The mean duration since diagnosis of DPN and back pain was 4.4 and 3.9 years, respectively, with patients with cancer-related pain being diagnosed on average 2.2 years before the study. Among those patients with back pain and cancer-related pain approximately twice as many patients reported having persistent pain than intermittent pain (Table 1). Mean baseline pain scores were consisted with patients having moderate pain, and mean sleep interference scores reflected moderate pain-related sleep interference. Concomitant analgesics were being taken by $37 \%$ to $57 \%$ of patients across the three disease groups. Few patients had been exposed to gabapentin in the past 14 days ( $\leq 11 \%$ in any group) and even fewer with DPN and cancerrelated pain were on concomitant gabapentin when pregabalin was commenced. In total $1.5 \%$ with back pain were taking concomitant gabapentin.

Most patients completed the 6-weeek treatment period (Table 2). Few patients $(\leq 3.4 \%)$ in any disease group discontinued due to lack of pregabalin efficacy and discontinuations due to adverse events were also relatively infrequent ( $\leq 6.1 \%$ across groups). The main reason for discontinuation was "other" which typically includes lost to follow-up and administrative reasons.

\section{Pregabalin Dosing}

The mean (SD) pregabalin dose in all patients with DPN was 229 (96) mg/day, for those on pregabalin monotherapy it was 228 (95) $\mathrm{mg} / \mathrm{day}$, and for those who added pregabalin to existing analgesics the dose was 231 (97) mg/day. Among patients with DPN, $10 \%$ took more than $300 \mathrm{mg} /$ day as their last dose (i.e. 450 or $600 \mathrm{mg} /$ day). For patients with back pain with a neuropathic component the mean (SD) pregabalin dose for all patients was 219 (95) $\mathrm{mg} /$ day, for those on pregabalin monotherapy it was 215 (97) $\mathrm{mg} /$ day, and for those who added pregabalin to existing analgesics the dose was 222 (94) mg/day. Among patients with back pain $9.8 \%$ took more than $300 \mathrm{mg} /$ day as their last dose. In patients with cancer-related neuropathic pain the mean (SD) pregabalin dose for all patients was 250 (121) $\mathrm{mg} / \mathrm{day}$, for those on pregabalin monotherapy it was 230 (115) $\mathrm{mg} / \mathrm{day}$, and for those who added pregabalin to existing analgesics the dose was 266 (124) mg/day. Among patients with cancer-related pain, $21 \%$ took more than $300 \mathrm{mg} /$ day as their last dose.

\section{EFFICACY}

The mean pain scores in each of the three disease groups decreased significantly from baseline over the course of the 6-week study (Fig. 1). The magnitude of the improvement was similar in each of the three groups, with the greatest improvement being observed at week 1, with further improvement thereafter until the week 6 endpoint. The magnitude of the pain at baseline, and the temporal improvement observed in all patients was similar in those treated with pregabalin monotherapy and those treated with pregabalin add-on therapy (data not shown). 

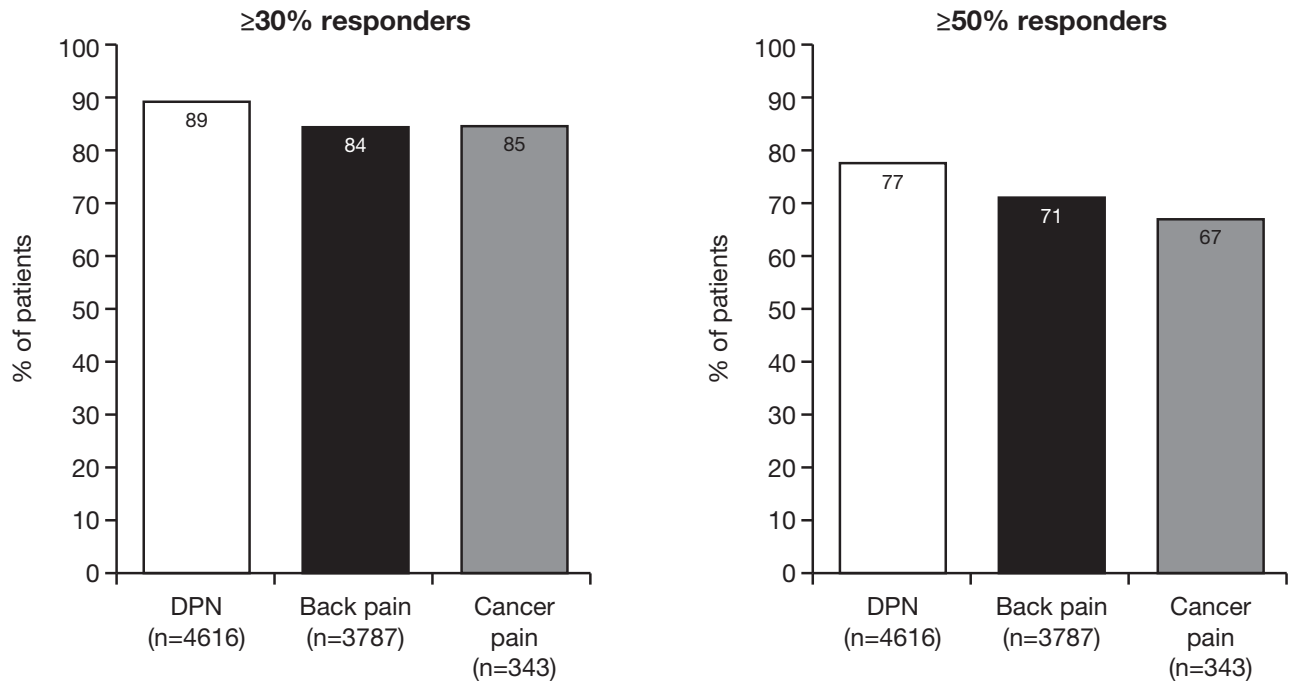

Fig. (2). Pain responder rates at 6-week endpoint (LOCF) Percentages of patients with $\geq 30 \%$, and $\geq 50 \%$, reduction in pain from baseline to endpoint (week 6).

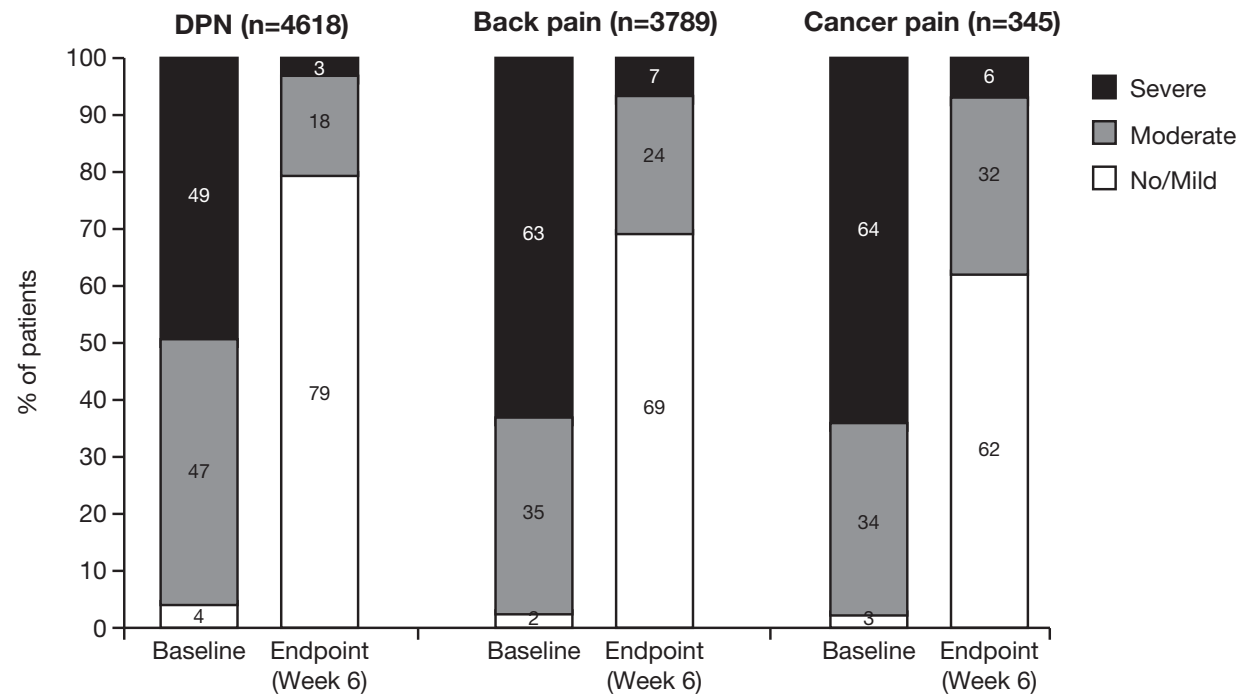

Fig. (3). Percentages of patients with mild, moderate and severe pain at baseline and 6-week endpoint (LOCF) Mild $=0-3$, moderate $=4-6$, severe $=7-10$

The mean (SE) change at endpoint in the pain score in the DPN, back pain and cancer-related pain groups, respectively, was $-3.9(0.03),-4.0(0.03)$, and $-3.8(0.11)$. These reductions represented improvements of $61 \%, 58 \%$, and $55 \%$, respectively. In the analysis of responder rates in all patients over $80 \%$ in each of the groups had a $\geq 30 \%$ pain reduction in their pain at 6 weeks, and over two-thirds had a $\geq 50 \%$ reduction (Fig. 2). In patients taking pregabalin monotherapy, the $\geq 30 \%$ responder rates were $92 \%, 89 \%$, and $84 \%$ in the DPN, back pain and cancer-related pain groups, respectively. The $\geq 50 \%$ responder rates were $81 \%, 79 \%$, and $71 \%$, respectively. In patients taking pregabalin add-on therapy the $\geq 30 \%$ responder rates were $85 \%, 81 \%$, and $85 \%$ in the DPN, back pain and cancer-related pain groups, respectively. The $\geq 50 \%$ responder rates were $70 \%, 65 \%$ and $64 \%$, respectively. The substantial improvement in pain was also evident in the change in the distribution of patients according to baseline pain severity (Fig. 3). In the DPN patients $49 \%$ had severe pain at baseline, as did $63 \%$ and $64 \%$ of those with back pain and cancer-related pain, respectively. At the week 6 endpoint very few patients in each of the groups $(\leq 7 \%)$ had severe pain and the majority reported mild or no pain.

In the evaluation of pain-related sleep interference, the mean change in each of the three disease groups was similar to the changes observed in the pain score, with significant improvements being observed from week 1 (Fig. 4A). The magnitude of the pain-related sleep interference at baseline, and the temporal improvement observed in all patients was similar in those treated with pregabalin monotherapy and those treated with pregabalin add-on therapy. The analysis of the severity of pain-related sleep interference at baseline and endpoint was also similar to the analysis of pain, with substantial percentages of patients reporting severe sleep interference at baseline and most reporting no or mild sleep interference at endpoint (Fig. 4B).

The PSQI was evaluated in a subset of patients. The improvement in the mean total score between baseline and endpoint was $45 \%$ in the DPN group, $47 \%$ in the back pain group and $44 \%$ in the cancer-related pain group (Table 3). The percentage of patients with a sleep disorder at baseline, 

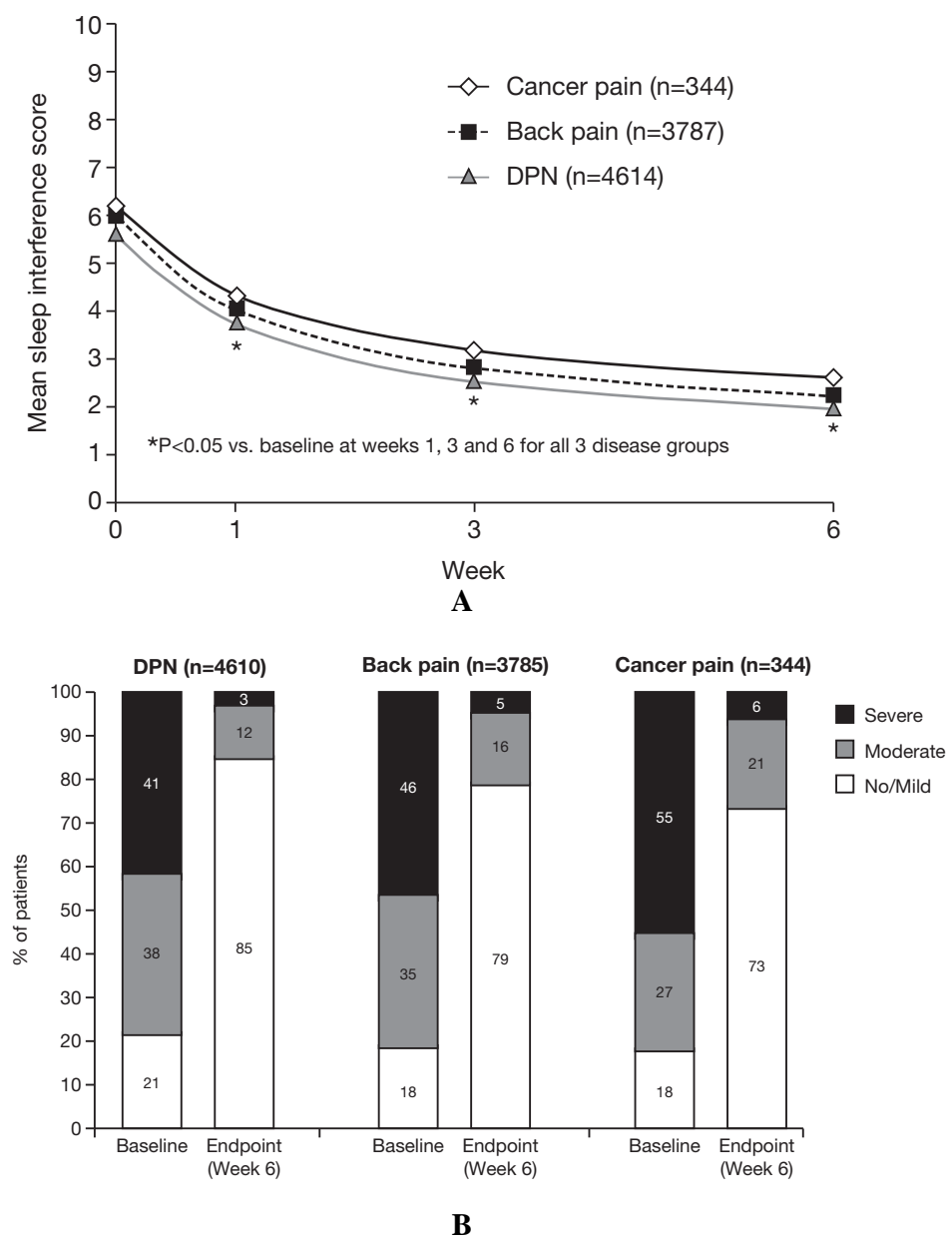

Fig. (4). (A) Mean sleep inference score and (B) percentages of patients with mild, moderate and severe sleep interference at baseline and week 6.

Each patient rated the extent to which pain interfered with sleep on an 11-point NRS from $0=$ not impaired to $10=$ severely impaired. $P$ values for change from baseline based on paired t- test.. Mild $=0-3$, moderate $=4-6$, severe $=7-10$.

Table 3. Summary of Pittsburgh Sleep Quality Index Results at Baseline and Week 6 Endpoint

\begin{tabular}{|l|c|c|c|}
\hline & DPN & Back Pain with Neuropathic Component & Cancer-Related Neuropathic Pain \\
\hline \hline Mean (SD) score & $(\mathrm{n}=775)$ & $(\mathrm{n}=587)$ & $(\mathrm{n}=63)$ \\
\hline Baseline & & & $10.6(5.0)$ \\
\hline Week 6 & $10.0(4.8)$ & $5.6(3.9)$ & $6.7(3.8)$ \\
\hline $\begin{array}{l}\text { Bad sleeper/sleep disorder, } \\
(\%)\end{array}$ & $5.4(3.3)$ & & $5.1)$ \\
\hline Baseline & & $469(80)$ & $55(87)$ \\
\hline Week 6 & $605(78)$ & $240(41)$ & $36(57)$ \\
\hline
\end{tabular}

PSQI total score ranges from $0-21$

Sleep disorder: PSQI total score $>5$

based on having a PSQI total score $>5$, was high in all disease groups, and highest $(87 \%)$ in the cancer-related pain group. In all three groups there was a substantial reduction from baseline in the percentages of patients classified as having a sleep disorder at week 6 (Table 3 ).

\section{Tolerability}

The most common adverse events are summarized in Table 4. The nature and frequency of adverse events was low in all three disease groups, with adverse events being reported most frequently in patients with cancer-related pain $(10.4 \%)$ and least in patients with DPN (5.4\%). The most common adverse events were dizziness, fatigue and somnolence.

\section{Satisfaction and Well-Being}

In the global evaluation of satisfaction with the tolerability of pregabalin, the overwhelming majority of 
Table 4. Overall Frequency of Adverse Events and Those Adverse Events Reported in $\geq 1 \%$ of Patients within Any of the Three Disease Categories

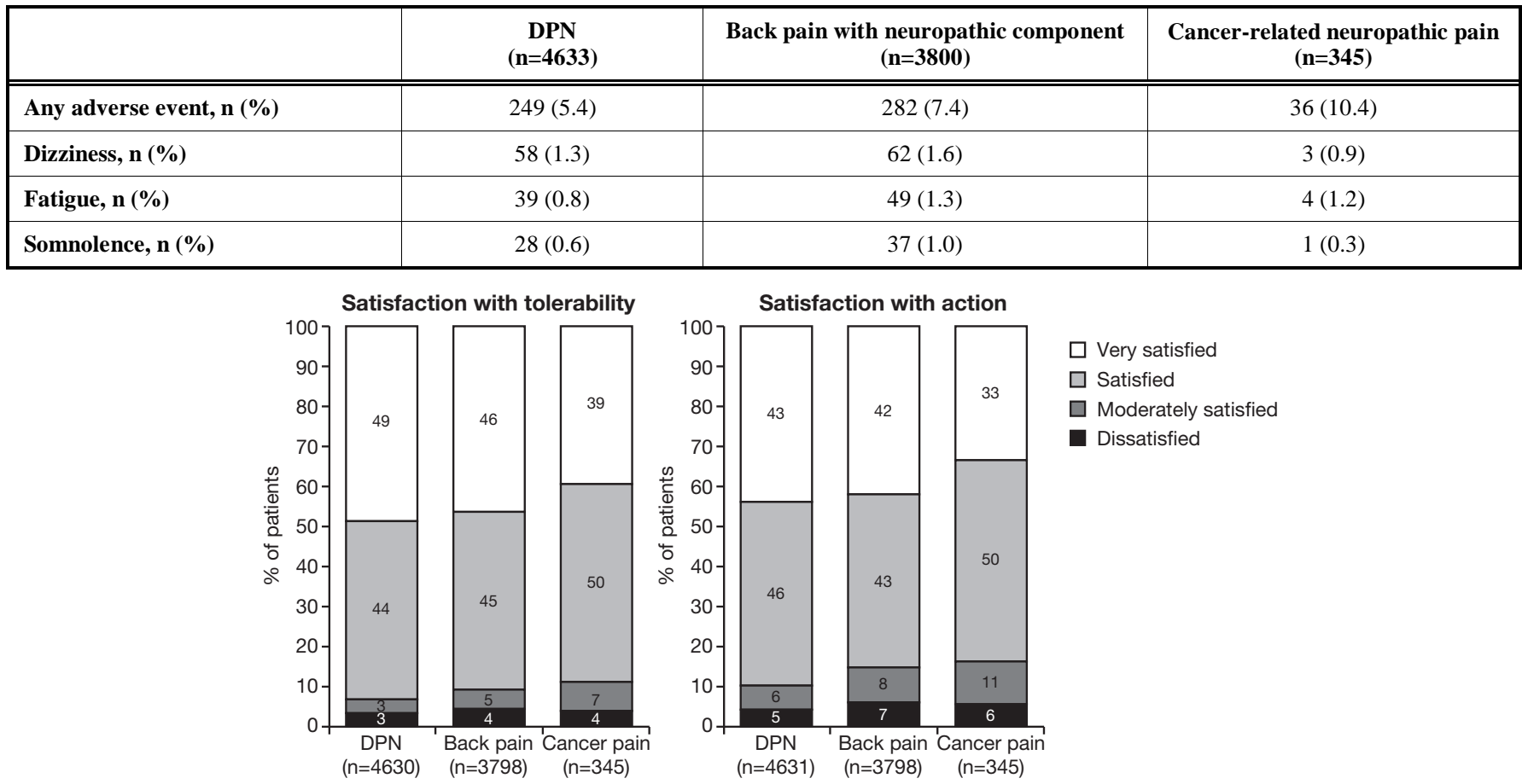

Fig. (5). Patient rating of satisfaction with the tolerability of pregabalin and the action of pregabalin.

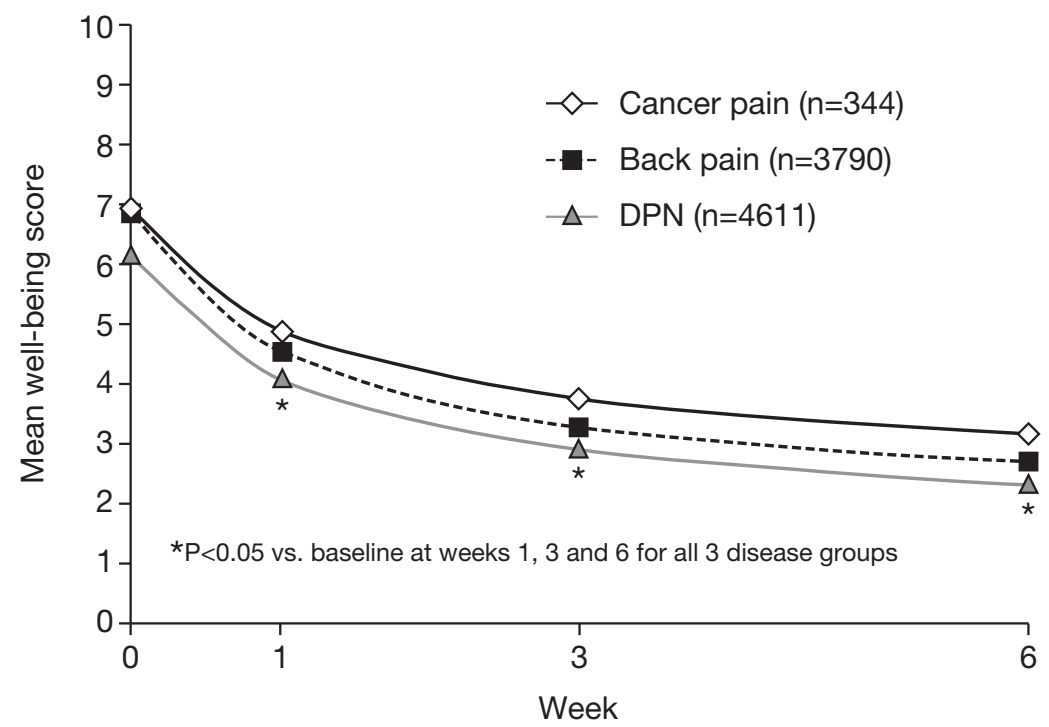

Fig. (6). Mean general well-being impairment score

Each patient rated if they experienced any impairments in general well-being in the last week on an 11-point NRS from 0=not impaired to $10=$ severely impaired. $\mathrm{P}$ values for change from baseline based on paired $\mathrm{t}$ - test.

patients in each disease group said they were either very satisfied or satisfied (Fig. 5). Most patients also rated themselves as being very satisfied or satisfied with the action of pregabalin at endpoint (Fig. 5). In the evaluation of general well-being there was a notable and significant improvement from baseline to endpoint in the level of impairment reported by patients in all three disease groups (Fig. 6). The rate of improvement was greatest in the first week, with further improvement observed until the 6 week endpoint. In the specific domains of well-being, there were dramatic increases between baseline and the week 6 endpoint in the percentages of patients in each of the disease groups who rated themselves as being at last often "calm and relaxed" and "full of energy", and a notable decrease in the percentages who felt "discouraged and sad" (Table 5).

\section{DISCUSSION}

This 6-week, open-label, observational study in a large sample of patients with neuropathic pain afforded the opportunity to evaluate pregabalin in day-to-day clinical practice in Germany. Pregabalin has undergone rigorous 
Table 5. Percentages of Patients Rating Well-Being in the Past Week as Always, Most of the Time, or Fairly Often at Baseline and Week 6

\begin{tabular}{|l|c|c|c|}
\cline { 3 - 4 } & DPN & Back Pain with Neuropathic Component & Cancer-Related Neuropathic Pain \\
\hline \hline Calm and Relaxed & $\mathbf{( n = 4 5 9 9 )}$ & $\mathbf{( n = 3 7 7 6 )}$ & $(\mathbf{n = 3 4 3 )}$ \\
\hline Baseline & $32.4 \%$ & $23.9 \%$ & $20.7 \%$ \\
\hline Week 6 & $82.6 \%$ & $77.5 \%$ & $63.3 \%$ \\
\hline Full of Energy & $(\mathbf{n = 4 5 8 9 )}$ & $\mathbf{( n = 3 7 6 4 )}$ & $(\mathbf{n = 3 4 2 )}$ \\
\hline Baseline & $17.2 \%$ & $11.8 \%$ & $8.5 \%$ \\
\hline Week 6 & $65.2 \%$ & $61.3 \%$ & $43.9 \%$ \\
\hline Discouraged and sad & $(\mathbf{n = 4 5 8 9 )}$ & $\mathbf{( n = 3 7 7 5 )}$ & $(\mathbf{n = 3 4 5 )}$ \\
\hline Baseline & $48.3 \%$ & $56.3 \%$ & $66.7 \%$ \\
\hline Week 6 & $16.7 \%$ & $18.2 \%$ & $31.0 \%$ \\
\hline
\end{tabular}

Well-being for each of the questions was rated as $1=$ always, $2=$ most of the time, $3=$ fairly often, $4=$ sometimes, $5=$ rarely, $6=$ never.

evaluation in RCTs and been shown to be effective and well tolerated in peripheral and central neuropathic pain [9-13]. In this observational study pregabalin appeared to be effective and well tolerated, satisfying to patients and associated with improved sleep and well-being.

As the study enrolled over 15,000 patients with peripheral neuropathic pain [29] we were able to identify subgroups of patients with neuropathic pain of specific aetiologies in sufficient numbers to enable analysis of these specific subgroups. Painful diabetic peripheral neuropathy is a debilitating complication of diabetes, being estimated to occur in $26-43 \%$ of patients with diabetes [31, 32]. It is considered a prototypical peripheral neuropathic pain state for the purposes of registration studies as it is considered to represent a "pure" neuropathic pain state not enmeshed with nociceptive pain. Back pain is common and low back pain is estimated to affect $50-85 \%$ of people at some point in their lives $[33,34]$ with $10-20 \%$ of people developing chronic low back pain [34]. In a survey of approximately 8000 people with low back pain in a variety of clinical settings application of the validated painDETECT screening tool identified $37 \%$ as having predominantly neuropathic pain [35]. Overall, it is estimated that $20-35 \%$ with low back pain will have distinct nociceptive and neuropathic components (i.e. mixed pain) and that at any given time approximately $6 \%$ of the general population will have low back pain with a neuropathic component [36]. Among patients with cancer the prevalence of pain has been reported to range from $14 \%$ to $100 \%$ and to occur in $50-70 \%$ of patients on treatment [37]. The causes of cancer pain are multifactorial and include infiltration of the tumour into tissues and structures, metastases, and the effects of treatment (surgery, radiation, chemotherapy) [37]. Cancer pain too can often be considered "mixed" pain as neuropathic and nociceptive components may be involved [7,8].

The baseline characteristics of the patients in each of the three disease groups were consistent with what one would expect to find in the clinical setting. Approximately twothirds of patients with back pain and cancer-related pain reported having persistent pain, a slightly higher proportion than among patients with DPN. The mean pain score at baseline was highest in patients with cancer and lowest in the DPN group, although in all three groups the mean pain scores were consistent with moderate to severe levels of pain. In fact almost half the patients with DPN and over $60 \%$ with back pain and cancer-related pain rated their pain as severe (score $\geq 7$ ) at baseline. The mean baseline pain and sleep interference scores in the DPN group in this sample was similar to that in a RCT of pregabalin in DPN [38], indicating that this clinical sample had symptoms as severe as a more rigorously derived research sample. Pregabalin was actually add-on therapy to other analgesics in over half the patients with back pain and cancer-related pain and in over one-third of those with DPN. Overall non-steroidal antiinflammatory drugs and cyclo-oxygenase- 2 inhibitors were the most commonly used concomitant analgesics, and were used least frequently in the DPN group. Narcotic analgesics and tricyclic antidepressants were used relatively infrequently, but use of both classes was highest in the cancer-related pain group and least in the DPN group probably reflecting the fact that the cancer-related pain was likely mixed pain and more patients had severe pain.

The mean reduction in pain from baseline to the 6 week endpoint in all three disease states was significant and of notable and clinically relevant magnitude ( $\geq 3.8$ points). The reductions were actually significant from the first assessment at week 1 with further reductions being observed through to week 6 . Given that pregabalin dosing was in accordance with the SmPC this means that at week 1 patients were taking $150-300 \mathrm{mg} / \mathrm{day}$. The robust mean reduction in pain at week 6 was reflected in the high responder rates; over $80 \%$ in each of the groups had a $\geq 30 \%$ pain reduction in their pain at 6 weeks, and over two-thirds had a $\geq 50 \%$ reduction. Striking was the finding that at baseline almost all patients had moderate or severe pain and at week 6 most patients had either mild or no pain. Even in the cancer-related pain group only $6 \%$ had severe pain at endpoint (vs. 64\% at baseline) and $62 \%$ actually had mild or no pain. The fact that so few patients discontinued the study due to lack of efficacy ( $\leq 3.4 \%$ across the groups) also indicates the pain relief was meaningful. The robustness of the pain relief in these patient subgroups was of similar magnitude to improvements observed in other smaller observational, 8-12-week studies of pregabalin in usual clinical settings in patients with neuropathic pain arising from a variety of underlying causes [39-41] and greater than in other observational studies in 
patients with refractory neuropathic pain $[42,43]$. The group with back pain in this study had a similar reduction in pain as patients with refractory radiculopathy in a 12-week, observational study [44].

The reduction in pain during pregabalin treatment was associated with a rapid, significant and marked reduction in pain-related sleep interference, a finding that is consistent across RCTs of pregabalin in neuropathic pain [9-13] and in other observational studies [40, 41]. In addition to assessing sleep interference, the present study also included an overall assessment of sleep quality using the PSQI which assesses several domains of sleep and daytime function in the previous month. The PSQI was not completed by all patients. This may be because the questionnaire was unfamiliar to the clinician and is quite long compared with the other assessments included in the study. Nonetheless there were sufficient questionnaires completed to provide meaningful data in the sub groups we examined. The mean baseline scores in the three disease groups in this analysis were similar to those of people with sleep disorders and people with depression examined in the initial validation study for the scale [30]. This indicates that overall sleep quality was poor in this sample and this was reflected also in the percentages of patients who had a PSQI score $>5$ at baseline ("bad sleepers"). Both the reduction in the mean PSQI score across the three groups at week 6 (44-47\%) and in the percentages of patients who were classed as "bad sleepers" from baseline to week 6 (35-40\% reduction), reflect a substantial and meaningful improvement in disturbed sleep. The substantial reduction in the percentages of patients with moderate or severe baseline sleep interference also reflects the meaningful improvement in sleep.

We noted that $<30 \%$ of patients in the sample we analysed were recorded as having a sleep disorder at the baseline visit by the treating physician. Yet the baseline sleep interference scale indicated that over $40 \%$ in each group had severe baseline sleep interference ( $80 \%$ moderate or severe) and the PSQI score indicated that 78$87 \%$ of patients across the three groups were classed as "bad sleepers". The inconsistency between the physician being aware of a sleep disorder and the findings of the systematic assessment may reflect the fact that sleep disturbance may be under-recognized or overlooked in patients with chronic pain. Given that disturbed sleep has a detrimental effect on function and quality of life $[45,46]$ and that disturbed sleep has been shown to increase pain sensitivity and reduce the pain threshold [47] it is important that both the pain itself and the disturbed sleep are targeted in the management of the patient.

This study also evaluated global patient-centred outcomes including satisfaction with treatment and general well-being. Such endpoints capture what we are ultimately trying to achieve in the clinic in managing patients such as those in the sample. The very high completion rate $(\geq 83 \%$ across groups) indicated patients found pregabalin treatment effective and well tolerated. The fact that most patients $(\geq 83 \%)$ rated themselves as either very satisfied or satisfied with the tolerability of pregabalin and very satisfied or satisfied with the action of pregabalin is further demonstration that pregabalin conferred meaningful benefits in these real-world patients. This was reflected in the marked and significant improvement in the general well-being scale score. Even at week 1 there was a significant improvement in well-being in all three disease states, reflecting the rapid improvement in pain and sleep interference.

Pregabalin appeared to be well tolerated in this study. This was not only apparent in the patient global evaluation of tolerability but also in the low frequency of adverse events. Among DPN patients 5.4\% reported adverse events with the highest rate being reported among patients with cancerrelated pain (10.4\%). Dizziness, fatigue and somnolence were the most commonly reported adverse events but were actually very infrequent $(\leq 1.6 \%)$. Dizziness and somnolence were the two most frequently reported adverse events in the RCTs [9-13], so although these events were infrequent in this study, the nature of the adverse events is consistent across studies. The very low rate of adverse events in this study may reflect the fact that pregabalin dosing was flexible and most patients were taking doses of $150-300 \mathrm{mg} / \mathrm{day}$ at the end of the 6-week treatment period, and not the higher doses which are associated with higher rates of adverse events. Being an open-label study both patients and physicians knew that pregabalin was the treatment and therefore what to expect in terms of adverse events. This too may have impacted the reporting of adverse events, even though they were proactively elicited by the physician.

The pregabalin dosing in this real-world group of patients was modest with the overall mean doses ranging from 219 $\mathrm{mg}$ /day in patients with back pain to $250 \mathrm{mg}$ /day in patients with cancer-related pain. The higher dose in patients with cancer-related pain likely reflects the more severe symptoms they had at baseline. The mean pregabalin doses in those on monotherapy and those who added pregabalin to existing analgesic therapy were not very different and nor was the actual change in pain scores.

It is germane to address some of the strengths and limitations of this open-label study. On one hand the study included a large number of patients in day-to-day clinical settings and pregabalin was dosed flexibly and used as either monotherapy or add-on therapy. Thus the findings should be generalizable to the wider population of patients with DPN, back pain with a neuropathic component and cancer-related pain. Observational studies are useful to address the fact that patients enrolled in RCTs, which are designed to test a null hypothesis and eliminate selection bias, may not be representative of the patient population at large. Observational studies go some way towards addressing the protestations from clinicians that "my patients are not like the patients in clinical trials" and provide decision makers with more pragmatic data to complement the rigorous data from RCTs. On the other hand, observational studies are thought to overestimate treatment effects, although there is evidence to the contrary [48, 49], and the lack of control group limits the interpretation of the results. A further limitation is that no specific tools or assessments were used to confirm the diagnosis of neuropathic pain made by the clinician. Thus, it is possible that patients with cancer-related pain and back pain without a neuropathic component might have been included although this would not have inflated the observed treatment effect. The study was only of 6 weeks duration which is relatively short given the chronicity of 
neuropathic pain and the duration for which patients had experienced symptoms.

In summary, this large observational study provided a means to evaluate pregabalin dosed as it is in clinical practice in patients with common, debilitating neuropathic pain conditions as they are present in day-to-day practice. Pregabalin, at doses generally of $300 \mathrm{mg} /$ day or less, was associated with rapid, significant and robust reductions in pain and pain-related sleep interference. The benefits on sleep were also reflected in the overall assessment of sleep quality, the PSQI, where marked improvements were observed and there was a notable reduction in the percentages of patients with disturbed sleep. High levels of patient-reported satisfaction in both the tolerability of and action of pregabalin indicate pregabalin treatment was agreeable to most patients. The significant improvement in well-being suggests that pregabalin treatment conferred meaningful benefit to patients. Overall the results complement the findings of RCTs of pregabalin in neuropathic pain, and other observational studies, and indicate pregabalin, either as monotherapy or as add-on therapy, has utility in the management of neuropathic pain associated with diabetic neuropathy, back pain and cancer.

\section{ACKNOWLEDGEMENTS AND DISCLOSURES}

The study was supported by Pfizer Pharma $\mathrm{GmbH}$, formerly of Karlsruhe (now Berlin), Germany. It was conducted between July 2004 and May 2005 before the requirement to register open-label studies online.

Roxana Varvara, Meryem Nimour, Birol Emir, and Matthias Brasser are all employees of divisions of Pfizer. Editorial assistance for the development of the manuscript was supported by Pfizer and provided by Janet Bray MRPharmS. Marie Ortiz and Sarah Bowen, employees of Pfizer, provided support for the data analysis. Thomas Toelle was not compensated for his work on the manuscript.

\section{CONFLICT OF INTEREST}

None declared.

\section{REFERENCES}

[1] Merskey H, Bogduk N, Eds. In: Classification of chronic pain: descriptions of chronic pain syndromes and definitions of pain Terms. $2^{\text {nd }}$ ed. Seattle, Washington: IASP Press 1994; pp. 209-12.

[2] Treede RD, Jensen TS, Campbell JN, et al. Redefinition of neuropathic pain and a grading system for clinical use: consensus statement on clinical and research diagnostic criteria. Neurology 2008; 70: 1630-5.

[3] Bouhassira D, Lanteri-Minet M, Attal N, Laurent B, Touboul C. Prevalence of chronic pain with neuropathic characteristics in the general population. Pain 2008; 136: 380-7.

[4] Torrance N, Smith BH, Bennett MI, Lee AJ. The epidemiology of chronic pain of predominantly neuropathic origin. Results from a general population survey. J Pain 2006; 7: 281-9.

[5] Freynhagen R, Bennett MI. Diagnosis and management of neuropathic pain. BMJ 2009; 339: b3002.

[6] Baron R, Binder A. [How neuropathic is sciatica? The mixed pain concept]. Orthopade 2004; 33(5): 568-75.

[7] Cherny NI, Thaler HT, Friedlander-Klar H, et al. Opioid responsiveness of cancer pain syndromes caused by neuropathic or nociceptive mechanisms: a combined analysis of controlled, singledose studies. Neurology 1994; 44(5): 857-61.

[8] Grond S, Radbruch L, Meuser T, Sabatowski R, Loick G, Lehmann KA. Assessment and treatment of neuropathic cancer pain following WHO guidelines. Pain 1999; 79(1): 15-20.
[9] Freeman R, Durso-Decruz E, Emir B. Efficacy, safety, and tolerability of pregabalin treatment for painful diabetic peripheral neuropathy: findings from seven randomized, controlled trials across a range of doses. Diabetes Care 2008; 31(7): 1448-54.

[10] Cappuzzo KA. Treatment of postherpetic neuralgia: focus on pregabalin. Clin Interv Aging 2009; 4: 17-23.

[11] Semel D, Murphy TK, Zlateva G, Cheung R, Emir B. Evaluation of the safety and efficacy of pregabalin in older patients with neuropathic pain: results from a pooled analysis of 11 clinical studies. BMC Fam Pract 2010; 11: 85

[12] van Seventer R, Bach FW, Toth CC, et al. Pregabalin in the treatment of post-traumatic peripheral neuropathic pain: a randomized double-blind trial. Eur J Neurol 2010; 17(8): 1082-9.

[13] Siddall PJ, Cousins MJ, Otte A, Griesing T, Chambers R, Murphy TK. Pregabalin in central neuropathic pain associated with spinal cord injury: a placebo-controlled trial. Neurology 2006; 67(10): 1792-800.

[14] Arnold L, Mease P, Silverman S. Pregabalin: an alpha2-delta (alpha2-delta) ligand for the management of fibromyalgia. Am J Manag Care 2010; 16 (5 Suppl): S138-43.

[15] Bandelow B, Wedekind D, Leon T. Pregabalin for the treatment of generalized anxiety disorder: a novel pharmacologic intervention. Expert Rev Neurother 2007; 7(7): 769-81.

[16] Beydoun A, Nasreddine W, Atweh S. Efficacy and tolerability of pregabalin in partial epilepsy. Expert Rev Neurother 2008; 8(7): 1013-24.

[17] Dooley DJ, Taylor CP, Donevan S, Feltner D. Ca2+ channel alpha2delta ligands: novel modulators of neurotransmission. Trends Pharmacol Sci 2007; 28(2): 75-82.

[18] Taylor CP, Angelotti T, Fauman E. Pharmacology and mechanism of action of pregabalin: the calcium channel alpha2-delta subunit as a target for antiepileptic drug discovery. Epilepsy Res 2007; 73(2): 137-50.

[19] Bauer CS, Nieto-Rostro M, Rahman W, et al. The increased trafficking of the calcium channel subunit alpha2delta-1 to presynaptic terminals in neuropathic pain is inhibited by the alpha2delta ligand pregabalin. J Neurosci 2009; 29(13): 4076-88

[20] Yuichi T, Keiko T, Hideki O, Mitsuo T. Pregabalin, S-(p)-3isobutylgaba, activates the descending noradrenergic system to alleviate neuropathic pain in the mouse partial sciatic nerve ligation model. Neuropharmacology 2007; 53: 842-53.

[21] Bockbrader HN, Wesche D, Miller R, Chapel S, Janiczek N, Burger P. A comparison of the pharmacokinetics and pharmacodynamics of pregabalin and gabapentin. Clin Pharmacokinet 2010; 49(10): 661-9.

[22] Johannessen LC, Patsalos PN. Drug interactions involving the new second- and third-generation antiepileptic drugs. Expert Rev Neurother 2010; 10(1): 119-40.

[23] Dworkin RH, O'Connor AB, Audette J, et al. Recommendations for the pharmacological management of neuropathic pain: an overview and literature update. Mayo Clin Proc 2010; 85(3 Suppl): S3-14.

[24] Attal N, Cruccu G, Baron R, et al. European Federation of Neurological Societies EFNS guidelines on the pharmacological treatment of neuropathic pain: 2010 revision. Eur J Neurol 2010; 17(9): 1113-e88.

[25] Bril V, England J, Franklin GM, et al. Evidence-based guideline: Treatment of painful diabetic neuropathy: Report of the American Academy of Neurology, the American Association of Neuromuscular and Electrodiagnostic Medicine, and the American Academy of Physical Medicine and Rehabilitation. Neurology 2011; 76(20): 1758-65.

[26] Portenoy RK. Treatment of cancer pain. Lancet 2011; 377(9784): 2236-47.

[27] Jost L, Roila F, ESMO Guidelines Working Group. Management of cancer pain: ESMO Clinical Practice Guidelines. Ann Oncol 2010; 21(Suppl 5): v257-60.

[28] European Medicines Agency. Pregabalin summary of product characteristics.

[29] Mallison R, Tilke C, Brasser M, Pittrow D. Efficacy and tolerability of pregabalin in patients with neuropathic pain. Observational study under clinical practice conditions. MMW Fortschr Med 2007; 149(14): 46.

[30] Buysse DJ, Reynolds CF 3rd, Monk TH, Berman SR, Kupfer DJ. The Pittsburgh Sleep Quality Index: a new instrument for psychiatric practice and research. Psychiatry Res 1989; 28(2): 193213. 
[31] Davies M, Brophy S, Williams R, Taylor A. The prevalence, severity, and impact of painful diabetic peripheral neuropathy in type 2 diabetes. Diabetes Care 2006; 29(7): 1518-22.

[32] Van Acker K, Bouhassira D, De Bacquer D, et al. Prevalence and impact on quality of life of peripheral neuropathy with or without neuropathic pain in type 1 and type 2 diabetic patients attending hospital outpatients clinics. Diabetes Metab 2009; 35(3): 206-13.

[33] Koes BW, van Tulder MW, Thomas S. Diagnosis and treatment of low back pain. BMJ 2006; 332(7555): 1430-4.

[34] Andersson GB. Epidemiological features of chronic low-back pain. Lancet 1999; 354(9178): 581-5.

[35] Freynhagen R, Baron R, Gockel U, Tölle TR. Pain DETECT: a new screening questionnaire to identify neuropathic components in patients with back pain. Curr Med Res Opin 2006; 22(10): 191120.

[36] Freynhagen R, Baron R. The evaluation of neuropathic components in low back pain. Curr Pain Headache Rep 2009; 13(3): 185-90.

[37] Christo PJ, Mazloomdoost D. Cancer pain and analgesia. Ann N Y Acad Sci 2008; 1138: 278-98.

[38] Tölle T, Freynhagen R, Versavel M, Trostmann U, Young JP Jr. Pregabalin for relief of neuropathic pain associated with diabetic neuropathy: a randomized, double-blind study. Eur J Pain 2008; 12(2): 203-13.

[39] Navarro A, Saldaña MT, Pérez C, Torrades S, Rejas J. Patientreported outcomes in subjects with neuropathic pain receiving pregabalin: evidence from medical practice in primary care settings. Pain Med 2010; 11 (5): 719-31.

[40] Xochilcal MM, Castro EM, Guajardo-Rosas J, et al. A prospective, open-label, multicentre study of pregabalin in the treatment of neuropathic pain in Latin America. Int J Clin Pract 2010; 64(9): 1301-9.
[41] Anastassiou E, Iatrou CA, Vlaikidis N, et al. Impact of pregabalin treatment on pain, pain-related sleep interference and general wellbeing in patients with neuropathic pain: a non-interventional, multicentre, post-marketing study. Clin Drug Investig 2011; 31(6): 417-26.

[42] Freynhagen R, Grond S, Schüpfer G, et al. Efficacy and safety of pregabalin in treatment refractory patients with various neuropathic pain entities in clinical routine. Int J Clin Pract 2007; 61(12): 198996.

[43] Lampl C, Schweiger C, Haider B, Lechner A. Pregabalin as mono or add-on therapy for patients with refractory chronic neuropathic pain: a post-marketing prescription-event monitoring study. J Neurol 2010; 257(8): 1265-73.

[44] Saldaña MT, Navarro A, Pérez C, Masramón X, Rejas J. Patientreported-outcomes in subjects with painful lumbar or cervical radiculopathy treated with pregabalin: evidence from medical practice in primary care settings. Rheumatol Int 2010; 30(8): 100515.

[45] Krystal AD, Roth T. Introduction: Definitions, measurements, and management in insomnia. J Clin Psychiatry 2004; 65(Suppl 8): 5-7.

[46] Kyle SD, Morgan K, Espie CA. Insomnia and health-related quality of life. Sleep Med Rev 2010;14(1): 69-82.

[47] Pieh C, Popp R, Geisler P, Hajak G. Sleep and pain: a bidirectional relation?. Psychiatr Prax 2011; 38(4): 166-7.

[48] Concato J, Shah N, Horwitz RI. Randomized, controlled trials, observational studies, and the hierarchy of research designs. N Engl J Med 2000; 342(25): 1887-92.

[49] Benson K, Hartz AJ. A comparison of observational studies and randomized, controlled trials. N Engl J Med 2000; 342(25): 187886.

(C) Toelle et al.; Licensee Bentham Open.

This is an open access article licensed under the terms of the Creative Commons Attribution Non-Commercial License (http://creativecommons.org/licenses/by-nc/3.0/) which permits unrestricted, non-commercial use, distribution and reproduction in any medium, provided the work is properly cited. 